\title{
RENDEZVOUS WITH SENSITIVITY
}

\author{
ANIMA NAGAR
}

\begin{abstract}
Let $(X, d)$ be a compact metric space and $f: X \rightarrow X$ be a self-map. The compact dynamical system $(X, f)$ is called sensitive or sensitivity depends on initial conditions, if there is a positive constant $\delta$ such that in each non-empty open subset there are distinct points whose iterates will be $\delta$-apart at same instance. This dynamical property, though being a very weak one, brings in the essence of unpredictability in the system. In this article, we survey various sensitivities and some properties implied by and implying such sensitivities.
\end{abstract}

\section{INTRODUCTION}

Sensitive Dependence on Initial Conditions is widely understood as being the central idea of "chaos" and was popularized by the meteorologist Edward Lorenz through the so-called "butterfly effect"(1963). The word "sensitive dependence on initial conditions" was first used by D. Ruelle in 1977.

The foundational contributions to the general theory of "chaos" can be credited to Poincare, Birkhoff, Landau, Ruelle, Takens, Lorenz, Rossler, Bowen, May, Liapunov, Sinai, Sharkovsky and many others who have not been named here.

The systematization of the mathematical theory and many initial mathematical definitions of "Chaos" can be credited to James Yorke [14]. Another important concept has been the virtue of systems with every orbit dense in the space. This notion of "minimality" has had its widest exploration by Joseph Auslander [4].

We look into the concept of "sensitivity" which largely combines the view of "chaotic sets" due to Yorke combined with the notion of "minimality" due to Auslander.

We note here that at the same time as when Auslander and Yorke were into publishing their work on sensitivity, there was defined a parallel definition of sensitivity by John Guckenheimer in a very restricted sense. His approach was mainly restricted to special kind of interval maps [10]. We follow the general definition due to Auslander and Yorke.

2010 Mathematics Subject Classification: 37B05, 54H20.

Key words and phrases: minimality, sensitivity, equicontinuity, Auslander-Yorke dichotomy, chaos.

This article is dedicated to Joseph Auslander and James Yorke.

This article is based on a talk given at the International Workshop and Conference on Topology \& Applications, Cochin, INDIA, Dec. 2018. 


\section{Compact Dynamical Systems}

Definition 2.1. A pair $(X, f)$ is called a compact dynamical system, where $(X, d)$ is a compact metric space and $f: X \rightarrow X$ is a continuous surjection.

Here $\mathcal{O}(x)=\left\{f^{n}(x): n \in \mathbb{N}\right\}$ is called the orbit of the point $x$.

Definition 2.2. For a system $(X, f)$, a point $x_{0} \in X$ is called a fixed point if $f\left(x_{0}\right)=x_{0}$. And $y_{0} \in X$ is called a periodic point if there exists $n \in \mathbb{N}$ such that $f^{n}\left(y_{0}\right)=y_{0}$. The smallest such $n$ is called the period of $y_{0}$.

Definition 2.3. The $\omega$-limit set of a point $x \in X$ under $f$, denoted as $\omega(x)$, is the set of all limit points of $\mathcal{O}(x)$.

Definition 2.4. A point $x \in X$ is said to be non-wandering if for every neighbourhood $U$ of $x$ there is a $n \in \mathbb{N}$ such that $f^{n}(U) \cap U \neq \varnothing$. The set of all non-wandering points of $f$ is denoted as $\Omega(f)$.

Definition 2.5. For a system $(X, f)$, a point $x \in X$ is called recurrent when $x \in \omega(x)$, $i$. e. for every open set $U$ containing $x$ there exist $j \in \mathbb{N}$ such that $f^{j}(x) \in U$. Equivalently there is a sequence $n_{k} \nearrow \infty$ such that $f^{n_{k}}(x) \rightarrow x$.

Definition 2.6. A subset $E \subset X$ is called $f$-invariant if $f(E) \subseteq E$.

Note that $\omega(x)$ is a closed, non-empty, $f$-invariant set with $f(\omega(x))=\omega(x)$.

Definition 2.7. A minimal set is a non-empty closed invariant set, which contains no proper non-empty closed invariant subsets.

Equivalently, a nonempty set $M \subset X$ is minimal if, for each $x \in M$, the orbit closure $\overline{\mathcal{O}(x)}=M$.

Fixed points and periodic orbits are minimal sets. Zorn's lemma shows that in a compact dynamical system $(X, f)$ any nonempty closed $f$-invariant subsets of $X$ contain minimal subsets.

Definition 2.8. A point $x \in X$ is called an almost periodic point if its orbit closure $\overline{\mathcal{O}(x)}$ is a minimal set.

Note that an almost periodic point is always recurrent, and periodic points are always almost periodic.

Definition 2.9. A compact system $(X, f)$ is called topologically transitive if for every pair of nonempty open sets $U, V$ in $X$, there is a $n \in \mathbb{N}$ such that $f^{n}(U) \cap V \neq \varnothing$ [Equivalently, $\left.U \cap f^{-n}(V) \neq \varnothing\right]$.

Proposition 2.1. For the compact system $(X, f)$, the following are equivalent:

(i) $(X, f)$ is topologically transitive.

(ii) There is an $x \in X$ with a dense orbit.

(iii) There is an $x \in X$ with $\omega(x)=X$. 
A point with a dense orbit is called a transitive point.

Note that a compact system is minimal when each of its points is a transitive point.

Definition 2.10. Let $(X, f)$ and $(Y, g)$ be compact systems. A continuous surjective map $\phi: X \rightarrow Y$ is called a equivariant map if $\phi(f x)=g \phi(x)$ for all $x \in X$.

In addition if $\phi$ is a homeomorphism, we call $\phi$ a conjugacy and say that $(X, f)$ and $(Y, g)$ are conjugate systems.

Note that

(1) If $x \in X$ is a periodic point, then $\phi(x)$ is a periodic point in $Y$.

(2) If $x \in X$ is a transitive point, then $\phi(x)$ is a transitive point in $Y$.

(3) If $y \in Y$ is a periodic point and $\phi$ is a conjugacy then $\phi^{-1}(y)$ is a periodic point in $X$.

(4) If $y \in Y$ is a transitive point and $\phi$ is a conjugacy then $\phi^{-1}(y)$ is a transitive point in $X$.

Note that for a periodic point $y \in Y$, and an equivariant map $\phi: X \rightarrow Y, \phi^{-1}(y)$ need not contain any periodic point in $X$. Same holds for transitive points. A simple example can be a union of minimal systems collapsing to a point, however we can say something concrete for almost periodic points.

Proposition 2.2. Let $\phi: X \rightarrow Y$ be a continuous surjection where $(X, f)$ and $(Y, g)$ are compact systems. If $y_{0} \in Y$ is almost periodic then $\phi^{-1}\left(y_{0}\right) \subset X$ contains an almost periodic point in $X$.

Proof. Let $y_{0} \in Y$ is almost periodic. Then $M_{0}=\overline{\mathcal{O}\left(y_{0}\right)} \subset Y$ is minimal. $\phi^{-1}\left(M_{0}\right)$ is a closed nonempty $f$-invariant subset of $X$ and so contains a minimal subset $N_{0}$. Now $\phi\left(N_{0}\right)=M_{0}$ and so there exists $x_{0} \in N_{0}$ such that $\phi\left(x_{0}\right)=y_{0}$. Thus $\phi^{-1}\left(y_{0}\right) \subset X$ contains an almost periodic point.

\section{Equicontinuity And Sensitivity}

Definition 3.1. Let $(X, f)$ be a compact dynamical system. A point $x \in X$ is said to be equicontinuous(stable) if, for any $\epsilon>0$, there is a $\delta>0$ such that $d\left(x, x^{\prime}\right)<\delta$ implies $d\left(T^{n}(x), T^{n}\left(x^{\prime}\right)\right)<\epsilon$, for $n=0,1,2, \ldots$

If all $x \in X$ are equicontinuous, then the system $(X, f)$ is called equicontinuous.

Definition 3.2. Thus $x \in X$ is sensitive(not stable / unstable) if and only if there is a $\delta>0$ such that there is a sequence $\left\{x_{j}\right\}$ in $X$ and $n \in \mathbb{N}$ such that $x_{j} \rightarrow x$ and $d\left(f^{n}(x), f^{n}\left(x_{j}\right)\right)>\delta$.

Equivalently, $(X, f)$ is sensitive at $x \in X$ if there is a $\delta>0$ such that for any $\epsilon>0$ there exists $y \in X$ with $d(x, y)<\epsilon$ and $n \in \mathbb{N}$ with $d\left(f^{n}(x), f^{n}(y)\right)>\delta$.

And if all $x \in X$ are sensitive, then the system $(X, f)$ is called sensitive or sensitively dependent on initial conditions.

Remark 3.1. Note that the uniform $\delta$ will follow due to compactness.

We note that if $x \in X$ is sensitive then there will be infinitely many such $n \in \mathbb{N}$ for which the above holds. 
Proposition 3.1. [5] Let $(X, f)$ be a compact system.

(i) If $x \in X$ is $\epsilon$-sensitive, then every point of $\overline{\mathcal{O}(x)}$ is $\epsilon / 2$ sensitive.

(ii) If $(X, f)$ is minimal and contains a sensitive point, then there is an $\epsilon>0$ such that every point is $\epsilon$-sensitive.

We note that this follows easily by triangular inequality and the fact that points in the orbit of sensitive points are sensitive.

Remark 3.2. The above gives a nice dichotomy result - all minimal systems are either equicontinuous or sensitive [5]. This is generally called the Auslander-Yorke dichotomy.

We look into some examples:

Example 3.1. Let $X=[0,1]$, the unit interval. Define $f: X \rightarrow X$ as

$$
f(x)= \begin{cases}2 x, & 0 \leq x \leq 1 / 2 ; \\ 2(1-x), & 1 / 2 \leq x \leq 1 .\end{cases}
$$

Then for the system $(X, f)$, if $|x-y|<\frac{1}{2^{n+1}}$, then $\left|f^{n}(x)-f^{n}(y)\right|=2^{n}|x-y|<\frac{1}{2}$ giving a sensitive system.

Example 3.2. Let $\mathcal{A}$ be a finite set and define $X=\mathcal{A}^{\mathbb{N}}$. Equip $X$ with product topology. Then $X$ is a compact metrizable space. One of the compatible metrics on $X$ is:

$$
d(x, y)=\inf \left\{\frac{1}{k+1} \mid x(n)=y(n) \text { for }|n|<k\right\} .
$$

Define $\sigma: X \rightarrow X$ by $\sigma(x)(n)=x(n+1)$. Then $\sigma$ is a continuous self map of $X$ - giving a compact system $(X, \sigma)$.

For any configuration $x=x_{0} x_{1} x_{2} \ldots \in X$, and $\epsilon>0$ there exists a configuration $y=$ $y_{0} y_{1} y_{2} \ldots \in X$ such that $d(x, y)<\epsilon$ but there is an $n \in \mathbb{N}$ for which $d\left(\sigma^{n}(x), \sigma^{n}(y)\right)>1 / 2$ giving a sensitive system.

Example 3.3. We recall Example 3.2. Let $\Lambda=\{0,1\}$ and define $X=\Lambda^{\mathbb{Z}}$. We consider the shift map $\sigma: X \rightarrow X$.

We obtain a minimal subset of $X$, by constructing an almost periodic point $p \in X$ since then $\overline{\mathcal{O}(p)}$ will be minimal. We take the classical construction due to Marston Morse and Axel Thue, giving the Morse-Thue sequence.

This construction is done using substitution: $0 \rightarrow 01,1 \rightarrow 10$. Hence,

$$
0 \rightarrow 01 \rightarrow 0110 \rightarrow 01101001 \rightarrow 0110100110010110 \rightarrow \cdots
$$

This will finally converge to some $x \in\{0,1\}^{\mathbb{N}}$. This construction indicates that every finite word in $x$ occurs syndetically often. Extend $x$ to $p \in X$ by defining $p(n)= \begin{cases}x(n), & n \geq 1 ; \\ x(-n-1), & n<0\end{cases}$

Every word in $p$ occurs syndetically and $p$ is symmetric at the mid point, and so $p$ is almost periodic. Thus $(\overline{\mathcal{O}(p)}, \sigma)$ is a minimal dynamical system.

It is easy to note that the system $(\overline{\mathcal{O}(p)}, \sigma)$ is sensitive.

Example 3.4. Let $X=\mathbb{T}=\{z \in \mathbb{C}:|z|=1\}$ be the unit circle. And let $g: X \rightarrow X$ be defined as $g(\theta)=\theta+2 \pi \alpha(\bmod 2 \pi)$, where $\alpha$ is an irrational number. Then $g$ is an isometry and $(X, g)$ is a minimal equicontinuous system. 


\section{Sensitivity And Chaos}

As discussed earlier, sensitivity plays an important role in "Chaos". The (possibly)second mathematical definition of chaos is given as,

Definition 4.1. [5] [A A slander and Yorke Chaos] The compact system $(X, f)$ is said to be chaotic if it is "topological transitive" and "pointwise sensitive".

Since "pointwise sensitive" is equivalent to "sensitivity" and "topological transitivity" is equivalent to the "existence of dense orbit", for a compact system Auslander-Yorke Chaos can be defined as a sensitive system with a dense orbit.

Remark 4.1. We note that this definition of Chaos makes sense even for non-compact systems.

Historically, this definition of Chaos did not catch any attention. It was a modification of this definition given a few years later that caught the fancy of the entire mathematical fraternity.

Definition 4.2. [7] [Devaney Chaos] A compact system $(X, f)$ is called Devaney Chaotic if:

1. $(X, f)$ is topologically transitive.

2. $(X, f)$ is sensitive.

3. $(X, f)$ admits a dense set of periodic points.

Thus by adding the condition of dense set of periodic points, Auslander-Yorke Chaos became Devaney Chaos and a well accepted definition too. But all this came with a price. Since a few years later a redundancy was reported in this definition. It was deduced that transitivity along with the denseness of periodic orbits actually implies sensitivity, giving a redundancy proved in [6, 18, 9] - where in [6, 18] the space is not even assumed to be compact.

Infact, there are various conditions under which transitivity implies sensitivity. We refer to [17] for a survey on this.

Despite this redundancy, sensitivity continues to be an integral part of chaos. Proving a system to be sensitive is much easier than proving it to be Devaney Chaotic.

It becomes meaningful at this juncture to talk about the first mathematical definition of Chaos given by $\mathrm{Li}$ and Yorke [14], which did generate a lot of excitement among the mathematical fraternity.

Definition 4.3. For the system $(X, f)$, the pair $(x, y) \in X \times X$ is called proximal if $\liminf _{n} d\left(f^{n}(x), f^{n}(y)\right)=0$, asymptotic if $\lim _{n} d\left(f^{n}(x), f^{n}(y)\right)=0$, not $\delta$-asymptotic if $\limsup d\left(f^{n}(x), f^{n}(y)\right)>\delta, \boldsymbol{L i}$-Yorke if $(x, y)$ is proximal but not asymptotic and $\delta$-LiYorke if $(x, y)$ is proximal but not $\delta$-asymptotic.

Definition 4.4. [14] A set $S \subseteq X$ containing at least two points is called a scrambled set if for any $x, y \in S$ with $x \neq y$, the pair $(x, y)$ is Li-Yorke. 
This means that for any two distinct $x, y \in S$, the orbits of $x$ and $y$ get arbitrarily close to each other but infinitely many times they are at a positive distance.

Definition 4.5. [14] [Li-Yorke Chaos] The system $(X, f)$ is called Li-Yorke chaotic if it has an uncountable scrambled set.

Later it was proved in [13] that Devaney chaos implies Li-Yorke chaos. Actually Li-Yorke chaos follows from transitivity and the existence of just one periodic orbit.

\section{Different Versions of Sensitivity}

For any nonempty, open $U \subset X$ for the system $(X, f)$ we define the seperating times:

$$
N_{\delta}(U) \quad=\left\{n \in \mathbb{N}: \operatorname{diam}\left(f^{n}(U)\right)>\delta\right\} .
$$

$(X, f)$ is sensitive if and only if there exists $\delta>0$ such that $N_{\delta}(U) \neq \varnothing$, for each nonempty, open $U \subset X$.

Recall that $S \subseteq \mathbb{N}$ is called thick if for each $k \in \mathbb{N}$ there exists $n_{k} \in \mathbb{N}$ such that $\left\{n_{k}, n_{k}+\right.$ $\left.1, \ldots, n_{k}+k\right\} \subset S$, is called cofinite if it the complement of a finite set in $\mathbb{N}$, is called syndetic if there exists $M \in \mathbb{N}$ such that $S \cap\{n, n+1, \ldots, n+M\} \neq \varnothing$ for each $n \in \mathbb{N}$, piecewise syndetic if it is the intersection of a syndetic set with a thick set, thickly syndetic if it has nonempty intersection with every piecewise syndetic set and is an IP-set if it contains the finite sum of its elements.

Stronger forms of sensitivity have been mainly studied by Christophe Abraham, Gerard Biau and Benoyt Cadre [1, Ethan Akin and Sergiy Kolyada [3, T. K. Subrahmonian Moothathu [16], Heng Liu, Li Liao, and Lidong Wang [15], Xiangdong Ye and Tao Yu [19], Wen Huang, Sergiy Kolyada, and Guohua Zhang [12].

Definition 5.1. The compact system $(X, f)$ is called

(1) strongly sensitive if there exists $\delta>0$ such that $N_{\delta}(U)$ is cofinite for any nonempty, open $U \subset X[1]$.

(2) $\boldsymbol{L} \boldsymbol{i}$-Yorke sensitive if there exists $\delta>0$ such that every $x \in X$ is a limit of points $y \in X$ such that the pair $(x, y)$ is proximal but not $\delta$-asymptotic [3].

(3) thickly sensitive if there exists $\delta>0$ such that $N_{\delta}(U)$ is thick for any nonempty, open $U \subset X[16]$.

(4) syndetically sensitive if there exists $\delta>0$ such that $N_{\delta}(U)$ is syndetic for some $m \in \mathbb{N}$ and any nonempty, open $U \subset X[16]$.

(5) piecewise syndetically sensitive if there exists $\delta>0$ such that $N_{\delta}(U)$ is piecewise syndetic for any nonempty, open $U \subset X$ [11, 12, 19].

(6) thickly syndetically sensitive if there exists $\delta>0$ such that $N_{\delta}(U)$ is thickly syndetic for any nonempty, open $U \subset X$ [11, 12, 15, 19].

(7) multi-sensitive if there exists $\delta>0$ such that $N_{\delta}\left(U_{i}\right) \neq \varnothing$ for any finite collection $U_{1}, \ldots, U_{k}$ of nonempty, open subsets of $X[16]$. 
(8) IP sensitive if there exists $\delta>0$ such that $N_{\delta}(U)$ is IP for any nonempty, open $U \subset X$ [12, 19].

Example 5.1. Recall Example 3.1. Here for any open $U \subset[0,1]$, there exists an $n \in \mathbb{N}$ such that $f^{n}(U)=[0,1]$. Hence, it is easy to see that this system is strongly sensitive.

Example 5.2. Recall Example [3.2. Here for any pair of open $U, V \subset X$, there exists an $N \in \mathbb{N}$ such that $\sigma^{n}(U) \cap V \neq \varnothing$ for all $n \geq N$. Thus it is easy to see that this system is thick sensitive and multi-sensitive.

Example 5.3. Recall Example 3.3. This system is sensitive and minimal. It is easy to see that it is syndetically sensitive.

From the definition, it follows that

$$
\text { cofinite sensitivity } \Rightarrow \text { thickly syndetical sensitivity } \Rightarrow \begin{gathered}
\text { thick sensitivity } \\
\text { syndetical sensitivity }
\end{gathered} \Rightarrow \text { sensitivity. }
$$

and

thickly syndetical sensitivity $\Rightarrow$ multi-sensitivity $\Rightarrow$ thick sensitivity $\Rightarrow$ sensitivity $\Leftarrow$ Li-Yorke sensitivity.

We refer to [3, 12, 15, 16, 19] for more examples on different kinds of sensitivity.

Infact, more can be said

Theorem 5.1. [3] Let $(X, f)$ be Li-Yorke sensitive then it is sensitive. If $(X, f)$ is sensitive and has a fixed point which is its unique minimal subset then it is Li-Yorke sensitive.

Theorem 5.2. [16] For interval systems and subshifts of finite type the notions of sensitivity and strong sensitivity are equivalent.

Theorem 5.3. [12, 16] A multi-sensitive system is also thickly sensitive. Moreover if the system is transitive, then the converse also holds.

Theorem 5.4. [19] For minimal systems the notions of thick sensitivity, thickly syndetic sensitivity, multi-sensitivity and IP sensitivity are all equivalent.

This is also equivalent to the fact that the system is not an almost one-to-one extension of its maximal equicontinuous factor.

Remark 5.1. Example 3.2 is thickly sensitive, thickly syndetic sensitive, multi-sensitive and IP sensitive.

This leads to considering sensitivity for different families of subsets of $\mathbb{Z}_{+}$.

Let $\mathfrak{P}=\mathfrak{P}\left(\mathbb{Z}_{+}\right)$be the collection of all subsets of $\mathbb{Z}_{+} \cdot \mathfrak{F} \subset \mathfrak{P}$ is called a family if it is hereditary upwards, i.e. if $F_{1} \subset F_{2}$ and $F_{1} \in \mathfrak{F}$ implies $F_{2} \in \mathfrak{F}$. A family $\mathfrak{F}$ is proper if it is a proper subset of $\mathfrak{P}$, i.e. it is neither empty nor whole of $\mathfrak{P}$. If a proper family $\mathfrak{F}$ is closed under finite intersection, then $\mathfrak{F}$ is called a filter. We refer to [8] for more details on this.

Definition 5.2. Let $\mathfrak{F}$ be a family. $(X, f)$ is $\mathfrak{F}$-sensitive if there is $\delta>0$ such that for any nonempty, open $U \subset X, N_{\delta}(U) \in \mathfrak{F}$. 
Proposition 5.1. [19] Let $\pi:(X, f) \rightarrow(Y, g)$ be an almost-open factor map between two compact systems, and let $\mathfrak{F}$ be a family. If $(Y, g)$ is $\mathfrak{F}$-sensitive then so is $(X, f)$.

$\mathfrak{F}$-sensitivity for various families $\mathfrak{F}$ have been studied in [19].

\section{Auslander-Yorke Dichotomy}

Recall the Auslander-Yorke dichotomy and note the following:

Proposition 6.1. [5] If the compact system $(X, f)$ is equicontinuous and topologically transitive, then $(X, f)$ is minimal, and $T$ is a homeomorphism.

Thus topologically transitive systems if equicontinuous are minimal and the dichotomy applies. However, not all topologically transitive systems are equicontinuous. These systems are then non minimal. But, a very natural question can be if we can derive Auslander-Yorke dichotomy kind of results for systems not minimal or a stronger version of such a dichotomy.

Recall that if the compact system $(X, f)$ is transitive but not minimal then the set of non-transitive points is dense.

Definition 6.1. A topologically transitive system is called almost equicontinuous if there is at least one equicontinuity point.

We note this dichotomy due to Akin, Auslander and Berg,

Proposition 6.2. [3] A transitive system is either sensitive or almost equicontinuous.

Definition 6.2. A system $(X, f)$ is called uniformly rigid if there exists a sequence $\left\{n_{k}\right\}$ in $\mathbb{N}$ such that the sequence $f^{n_{k}}$ tends uniformly to the identity map on $X$.

We note this dichotomy due to Glasner and Weiss,

Proposition 6.3. [9] A topologically transitive system without isolated points which is not sensitive is uniformly rigid.

Definition 6.3. [11, 12] The point $x \in X$ is called syndetically equicontinuous for the system $(X, f)$, if there exists a neighborhood $U \ni x$ such that $\left\{n \in \mathbb{N}:\left\{f^{n}\right\}\right.$ is equicontinuous at $x\}$ is a syndetic set.

Note that the set of syndetically equicontinuous points for $(X, f)$ is a superset of the set of equicontinuity points of $(X, f)$ and that if $(X, f)$ is thickly sensitive then it cannot contain any syndetically equicontinuous points.

Definition 6.4. [11, 12] The system $(X, f)$ is syndetically equicontinuous if every $x \in X$ is syndetically equicontinuous.

Example 6.1. Recall Example 3.4. This system is equicontinuous and minimal. It is easy to see that it is syndetically equicontinuous.

Proposition 6.4. [11, 12] If $(X, f)$ is an almost one-to-one extension of a minimal equicontinuous system, then it is syndetically equicontinuous. 
Proposition 6.5. [12] Let $\pi:(X, f) \rightarrow(Y, g)$ be an almost one-to-one factor map between minimal systems. Then $(X, f)$ is syndetically equicontinuous if and only if $(Y, g)$ is also.

We note this dichotomy due to Huang, Kolyada and Zhang,

Theorem 6.1. [12] Let $(X, f)$ be a transitive system. Then either $(X, f)$ is thickly sensitive, or every transitive point is syndetically equicontinuous. In particular, if $(X, f)$ is minimal, then it is either thickly sensitive or syndetically equicontinuous.

Remark 6.1. Note that in the absence of transitivity the above need not hold. Refer to [12] for a counterexample.

Some more Auslander-Yorke dichotomy type theorems are obtained in [19] involving $\mathfrak{F}$-sensitivity for various families $\mathfrak{F}$.

\section{The world awaits more such dichotomies!}

\section{REFERENCES}

[1] Christophe Abraham, Gerard Biau and Benoyt Cadre, Chaotic Properties of Mappings on a Probability Space, Journal of Mathematical Analysis and Applications, 266 (2002), 420-431.

[2] Ethan Akin, Joseph Auslander, Kenneth Berg, When is a transitive map chaotic?, in: Convergence in ergodic theory and probability, de Gruyter, Berlin-New York, 1996, 25-40.

[3] Ethan Akin and Sergiy Kolyada, Li-Yorke sensitivity, Nonlinearity 16(4) (2003), 1421-1433.

[4] Joseph Auslander, Minimal flows and their extensions, North-Holland Mathematics studies,153 (1988).

[5] Joseph Auslander and James Yorke, Interval maps, factors of maps, and chaos, Tohoku Mathematical Journal, Second Series, 32.2, (1980) 177-188.

[6] Banks, J. Brooks, J. Cairns, G. Davis, G. and Stacey, P. On Devaney's definition of chaos, Amer. Math. Monthly, 99, 332-334(1992).

[7] Robert Devaney, An introduction to chaotic dynamical systems, 2nd ed., Addison Wesley, (1989).

[8] Hillel Furstenberg, Disjointness in ergodic theory, minimal sets and a problem in diophantine approximation, Mathematical Systems theory, 1 (1967), 1-49.

[9] Eli Glasner and Benjamin Weiss, Sensitive dependence on initial conditions, Nonlinearity, 6, 10671075(1993).

[10] John Guckenheimer, Sensitive dependence to initial conditions for one dimensional maps, Comm. Math. Phys. 70, 133-160(1979).

[11] Wen Huang, Danylo Khilko, Sergiy Kolyada and Guohua Zhang, Dynamical compactness and sensitivity. J. Differential Equations 260(9) (2016), 6800-6827.

[12] Wen Huang, Sergiy Kolyada and Guohua Zhang, Analogues of Auslander-Yorke theorems for multisensitivity, Ergod. Th. \& Dynam. Sys. (2018), 38, 651-665

[13] Wen Huang, Xiangdong Ye, Devaney's chaos or 2-scattering implies Li-Yorke's chaos, Topology Appl. 117 (2002), No. 3, 259-272.

[14] Tien-Yien Li and James Yorke, Period three implies chaos, Am. Math. Month. 82(1975), 985-992.

[15] Heng Liu, Li Liao, and Lidong Wang, Thickly syndetical sensitivity of topological dynamical system, Discrete Dyn. Nat. Soc. 2014, Art. ID 583431, 4 pp.

[16] T. K. Subrahmonian Moothathu. Stronger forms of sensitivity for dynamical systems. Nonlinearity 20(9) (2007), 2115-2126.

[17] Anima Nagar and V. Kannan Topological Transitivity for Discrete Dynamical Systems, Applicable Mathematics In The Golden Age, Narosa Publications(2003), 534-584.

[18] Stephen Silverman, On maps with dense orbits and the definition of chaos, Rocky Mountain Jour. Math. 22, 353 - 375(1992). 
[19] X. Ye and Tao Yu. Sensitivity, proximal extension and higher order almost automorphy. Trans. Amer. Math. Soc. 370 (2018), 3639-3662.

Department of Mathematics, Indian Institute of Technology Delhi,, Hauz Khas, New DELHI 110016, INDIA

E-mail address: anima@maths.iitd.ac.in 